Article

\title{
Microscopic Observation Drug Susceptibility (MODS) Assay: A Convenient Method for Determining Antibiogram of Clinical Isolates of Mycobacterium tuberculosis in Ghana
}

\author{
Enid Owusu ${ }^{1, *(1)}$ and Mercy Jemima Newman ${ }^{2}$ \\ 1 Department of Medical Laboratory Sciences, School of Biomedical and Allied Health Sciences, University of \\ Ghana, P.O. Box LG 25, Accra, Ghana \\ 2 Department of Medical Microbiology, University of Ghana Medical School, University of Ghana, \\ P.O. Box LG 25, Accra, Ghana; mjnewmangh@gmail.com \\ * Correspondence: enidowusu115@yahoo.com or eowusu@chs.edu.gh; Tel.: +233-050-891-7569
}

Received: 20 November 2019; Accepted: 13 January 2020; Published: 25 January 2020

check for updates

\begin{abstract}
Background: Present methods for drug susceptibility tests (DST) rely on culture methods that are sophisticated and relatively faster, or a slow and cheaper option. These methods frustrate disease control; therefore, there is a need for methods that incorporate key functions of microscopy and culture, with reduced cost burden and sophistry. Thus, the purpose of this study was to identify which, among the most commonly used (in Ghana) methods, can conveniently be used at health centers located in rural areas for effective DST determination of Mycobacterium tuberculosis (MTB). (2) Methods: Mycobacterium tuberculosis isolates were tested for their susceptibility to streptomycin, isoniazid, rifampicin, ethambutol (SIRE), and pyrazinamide by microscopic observation drug susceptibility (MODS) and BACTEC MGIT 960 methods. Evaluations were based on shorter turnaround periods, rapidity, ease of use, cost, etc. A comparative analysis was statistically expressed as kappa values. (3) Results: Endpoints for drug susceptibilities by MODS averaged 13 days (7-32), whilst that for BACTEC MGIT 960 was 10 days with a further 12 days to detect resistance. Therefore, a turnaround period of 22 days was needed for DST by BACTEC MGIT 960, compared to 13 days for MODS. There were differences in correlation levels between the two methods, as determined by their kappa values. (4) Conclusion: The MODS assay was found to be less costly, more user-friendly, and still able to be conveniently used at health centers located in rural areas known to be endemic for TB, particularly in Ghana.
\end{abstract}

Keywords: MODS; Mycobacterium tuberculosis; susceptibility; tuberculosis

\section{Introduction}

Pulmonary tuberculosis (TB) is a preventable disease with a huge global impact due to its mode of transmission. It remains one of the world's leading causes of death, killing more than 2 million people every year [1-3]. According to the 2019 WHO Global Tuberculosis Report, geographically, most TB cases in 2018 were in the WHO regions of South-East Asia (44\%), followed by Africa, with $24 \%$ [4]. Earlier reports have indicated that more than $95 \%$ of cases and deaths due to TB occur in developing countries [3,5]. Therefore, the prevalence of the condition is reportedly relatively high in countries with limited resources, such as Ghana [3,5]. This problem could be attributed to the poor hygienic practices, unfavorable living conditions, and resource constraints in these areas. Comorbidity with HIV coupled with reports of emerging resistant TB strains (MDR-TB) has frustrated efforts at disease control [5-7]. Rapid detection of the disease coupled with faster methods for the determination of susceptibility to 
Mycobacterium tuberculosis (MTB) isolates to primary treatment antibiotics is a major step in efforts to control the disease. Various microbiological methods are available for the susceptibility determination of MTB to treatment with drugs/antibiotics, such as streptomycin, rifampicin, ethambutol, isoniazid, and pyrazinamide [8,9]. These drugs have been used effectively for several decades; however, the emergence of MTB strains showing varying levels of resistance to the drugs has frustrated efforts at TB control, a situation that is particularly associated with endemic countries with limited resources. This phenomenon is present in Ghana [5], where drug-resistant TB continues to pose a public health threat. In 2018, there were about half a million new cases of rifampicin-resistant TB (of which $78 \%$ had multidrug-resistant TB) [4].

Methods for determining drug susceptibility tests (DST) for MTB involve the use of an isolate culture in a drug-incorporated mycobacterial medium. Frequently used media include Lowenstein-Jensen (L-J) egg based, Middlebrook 7H11 solid media, and Middlebrook 7H9 liquid medium. Methods for DST determination include the automated BACTEC MGIT 960 broth culture system and the Lowenstein-Jensen solid culture assay. Among these culture methods, the automated BACTEC assay is the most sensitive; this approach is suitable for growing MTB even in AFB negative samples within relatively shorter detection periods (7-14 days) [10], though it is sophisticated and costly. The burden of cost makes this method inaccessible to peripheral healthcare points in TB endemic communities [10]. The L-J solid culture method is relatively simpler and cheaper, but it is slow, requiring longer culture periods (4-8 weeks) for DST determination. The period can be shortened with the use of Middlebrook and Cohn's solid media using agar [11]; however, its long turnaround time makes it unreliable, in addition to this media not being easily available in some locations. In the midst of these challenges, new cases of tuberculosis continue to be reported annually [4]. This situation, coupled with the emergence of MDR-TB, as well as comorbidity with infections such as HIV, tend to aggravate the situation [5-7].

The need for drug susceptibility methods that are cheap, rapid, convenient, and easily applicable in rural health posts of TB-endemic areas cannot be overemphasized. Such methods will provide useful information on suitable antibiotics and their effective therapeutic doses, as well as on the current prevalence of circulating MDR-TB in endemic areas. These efforts will contribute immensely to effective disease control. As emphasized by some researchers, a viable method is one that would incorporate functionally-suitable aspects of microscopy and culture, and also consider robustness, while being technically less demanding [12-14].

The microscopic observation drug susceptibility (MODS) assay is one such qualitative test which fits into these criteria. It involves the visualization of MTB growth in broth, in addition to typical cord (pellicle) formation. This method has been recommended by several studies as being relatively rapid, cost effective, and less technically demanding [15-19]. Its associated limitations have to do with interspecies differentiation, a challenge which can easily be overlooked, given the advantages derived from the rapid results obtained from this method [14]. The adaptation of the MODS assay can be helpful for the early determination of DST for TB, particularly in under-resourced areas. The sensitivity of the MODS method has been estimated to be about $94 \%$, while that of Lowenstein-Jensen (LJ) culture has been reported to be about $86.9 \%$ [15].

Though many tools exist for the diagnosis of MTB disease, they are limited by their inability to provide results on the status of drug resistance [16-23]. This study therefore purposes to identify which, among the most commonly used (in Ghana) methods, can conveniently be applied at health centers located in rural areas for effective DST determination of MTB isolates. Thus, the MODS and BACTEC MGIT 960 methods were evaluated for their usefulness in DST determination of MTB, using clinical MTB isolates. Specifically, the study sought to evaluate the feasibility of applying these methods in resource-constrained areas by evaluating their turnaround periods, rapidity, ease of use, and cost effectiveness. 


\section{Materials and Methods}

\subsection{Study Design, Setting and Subjects}

This was a cross-sectional study conducted at the chest clinic of the Korle-Bu teaching hospital, the premier public tertiary hospital and the largest referral hospital in Ghana. The study was carried out in a Class II biosafety cabinet in a biosafety level 3 (BSL3) laboratory. All safety precautions were appropriately observed. Subjects who consented to participate in the study were instructed on sputum collection, as recommended by the World Health Organization (WHO) [24]. Each patient was required to produce three sputum samples for the investigation, i.e., one on the spot, an early morning sample the next day, and a third one upon arrival at the laboratory. Some of the study participants could not produce all three samples, while samples which contained saliva instead of sputum were excluded. Thus, a total of 533 sputum samples were obtained from 221 clinically-suspected MTB patients who consented to be enrolled in the study. After initial screening using Zeihl-Neelsen smear microscopy, 130 samples with smears showing no acid fast bacilli (MTB) were excluded. Therefore, a total of 403 samples qualified to be used for the study. Out of the 403 samples, 54 participants decided to opt out of the study. Thus, a total of 349 samples were finally available for analyses. Ethical approval was given by the Scientific and Ethical review boards of Ministry of Health and the Korle-Bu Teaching hospital.

\subsection{Microbiological Analysis of Study Samples}

A minimum of one (1) and a maximum of three (3) sputum samples were obtained from each subject. These were processed to reduce fast growing bacteria contaminants by the $N$-acetyl-L-cysteine decontamination method [25]. The decontaminated sputum samples were then routinely analyzed by the Zeihl-Neelsen smear microscopy, and cultured on LJ media [26], as well as the MODS assay [14] and the BACTEC MGIT 960 (Becton Dickinson, Sparks, MD, USA) method [27] for MTB growth.

\subsection{Mycobacterium Tuberculosis Culture}

Sputum samples were decontaminated by the N-A-L-C-sodium hydroxide method as described in [25], pelleted by centrifugation (at 3,200 $\mathrm{g}$ for $20 \mathrm{~min}$ ), and resuspended in $1000 \mu \mathrm{L}$ of sterile saline. The turbidity of the suspension was adjusted to a McFarland number 1 standard and then used to inoculate for cultures, as well as DST by BACTEC MGIT 960 and direct MODS methods. The isolated cultures from processed samples were then used for the indirect MODS DST method. The procedures used were adapted from Caviedes et al. [17] and Musa et al. [28].

For culture on LJ media, $250 \mu \mathrm{L}$ of decontaminated bacterial suspension was inoculated. The LJ slants were incubated at $37{ }^{\circ} \mathrm{C}$ and examined twice a week for 42 days. Growth on the LJ slants was checked with the Zeihl-Neelsen smear microscopy (Sigma-Aldrich, St. Louis, MO, USA) for the presence of AFB. Growth was recorded at 28 days and at 42 days with the following descriptions: +++ for confluent growth, ++ for $>100$ colonies, and 1-100 actual numbers of colonies.

For culture with BACTEC MGIT 960,500 $\mu \mathrm{L}$ of bacterial suspension was inoculated in MGIT tubes and incubated at $37^{\circ} \mathrm{C}$ in the BACTEC MGIT 960 system, which monitors for fluorescence every day for up to 42 days by automated mechanisms.

With the MODS culture method, $130 \mu \mathrm{L}$ of decontaminated bacterial suspension was inoculated in Middlebook $7 \mathrm{H} 9$ broth and incubated at $37^{\circ} \mathrm{C}$ for 42 days. The wells were examined daily after day five under an inverted microscope for growth; bacterial growth was seen as granular serpentine cords. All negative cultures were further confirmed with Z-N microscopy after the 42 days' incubation.

\subsection{Drug Susceptibility Testing (DST)}

Drug susceptibility tests were performed by the BACTEC MGIT 960 susceptibility method, as well as the direct and indirect MODS methods. The procedures for BACTEC MGIT 960, as well as the direct and indirect MODS methods, are briefly described below. 


\subsubsection{BACTEC MGIT 960 Susceptibility Method}

Drug susceptibility tests set up by the BACTEC system required five tubes for the test drugs and two others for pyrazinamide (PZA). Streptomycin, isoniazid, rifampicin, and ethambutol (SIRE) were prepared in dilutions of 1:100 (1\%), whilst pyrazinamide (PZA) was diluted to 1:10 (10\%). Control tubes contained broth without drugs. Each test contained $7 \mathrm{~mL}$ of broth with $800 \mu \mathrm{L}$ of the supplement and $100 \mu \mathrm{L}$ of specified antibiotic. Then, $500 \mu \mathrm{L}$ of MTB inoculum was dispensed into each test tube. Control tubes contained no antibiotics, whiles 1:100 inoculum dilutions were set up as microbial controls; this was to ensure growth in the controls before tests. DST was performed for all positive MGIT on Isoniazid $0.2 \mu \mathrm{g} / \mathrm{mL}$, Streptomycin $4 \mu \mathrm{g} / \mathrm{mL}$, Rifampicin $40 \mu \mathrm{g} / \mathrm{mL}$, Ethambutol $2 \mu \mathrm{g} / \mathrm{mL}$, and Pyrazinamide $200 \mu \mathrm{g} / \mathrm{mL}$, according to WHO guidelines [29].

Tests and controls were arranged in the BACTEC MGIT 960 instrument for a 14-day incubation period. Tests with growth units of less than 200 were classified as indeterminate, and therefore, repeated. Controls usually attained 400 growth units before flagging for end point. Tests with fewer than 100 growth units were indicative of susceptibility. Smears were made from controls and Z-N stained to establish purity.

\subsubsection{Microscopic Observation Drug Susceptibility (MODS)}

The MODS susceptibility assay was conducted by the direct and indirect methods. The tests were done in a 12-well culture plates. Each test well contained $2 \mathrm{~mL}$ Middlebrook 7H9 broth, $200 \mu \mathrm{L}$ of suspension, and $34 \mu \mathrm{L}$ of the appropriate test antibiotic. A McFarland 0.5 (approximately $104 \mathrm{CFU} / \mathrm{mL}$ ) suspension of isolates was used. The controls were set up in the first row of the plate. For each plate, two antibiotics in combinations of streptomycin and isoniazid (S\&I) and rifampicin and ethambutol (R\&E) were set up with the control. The drug concentrations were similar for the BACTEC MGIT 960 method according to WHO guidelines [29].

Each plate was set up with a complement of a positive and a negative control in addition to the test wells. Tests for pyrazinamide were set up on a separate plate, as this required conditions that were different from those of the other test drugs, including a relatively low $\mathrm{pH}$ and so a higher dose of inoculum. Each PZA test well contained $2 \mathrm{~mL}$ of Middle brook 7H9 broth, $200 \mu \mathrm{L}$ of supplement, and $34 \mu \mathrm{l}$ of reconstituted PZA. All tests and controls were appropriately labeled.

Tests for the direct method were inoculated with $140 \mu \mathrm{L}$ of decontaminated sputum sample prepared in pelleted dilutions as indicated. An indirect test was inoculated with appropriate diluents of MTB colony suspension. A negative control well contained only MODS media. A positive control contained the MODS media and MTB inoculum at a dilution of 1:100 and 1:10 for PZA.

Plates were incubated at $37{ }^{\circ} \mathrm{C}$ and examined twice weekly after an initial daily check for contamination for up to 42 days. Detectable serpentine clusters (pellicle formation) of MTB growth observed under an inverted microscope at $40 \times$ magnification and confirmed by AFB microscopy was indicative of positive culture. The growth observed in both the drug-free and the drug-containing wells was indicative of resistance, whilst tests with growth in drug-free and no growth in drug-incorporated media indicated susceptibility of the test isolates to the test drugs [18]. If growth was detected in only one control well, MODS-DST was recorded as indeterminate for technical analysis

The broth from the MGIT tubes was poured into the MODS wells to find out whether cord formation in acidic medium could be easily visualized. The broth medium for the determination of DST for streptomycin, isoniazid rifampicin, and ethambutol (SIRE) was prepared in the laboratory. With respect to pyrazinamide testing, the quality could have been compromised if the media had been prepared in the laboratory.

\subsection{Statistical Analysis}

Data obtained from this study was stored and analyzed using the Microsoft office Excel 2007 software (Microsoft ${ }^{\circledR}$ Office Professional 2007, Microsoft Corporation, Redmond, WA, USA) and 
GraphPad software version 8.0. (GraphPad, La Jolla, CA, USA). The concordance of susceptibility results was determined based on the sensitivity, specificity, and positive and negative predictive values for the detection of resistance with $95 \%$ confidence intervals (CIs), as well as with kappa values [30]. A kappa coefficient $(\mathrm{K})$ value of $<0.4,0.4-0.6,0.61-0.8$, and $0.81-1.0$ indicated low agreement, moderate agreement, substantial agreement, and perfect agreement, respectively. A $p$-value less than 0.01 was considered statistically significant. For the estimations of the sensitivity, specificity, and predictive value of detection for the methods, a positive reference result was defined as a positive culture according to at least one method for which cross contamination had been conclusively ruled out. A negative reference result was defined as any sample in which all three culture methods yielded negative results, or when two were negative and the third indeterminate, owing to repeated bacterial or fungal overgrowth as defined in an earlier study [14].

\section{Results}

\subsection{Drug Susceptibility Testing}

A total of 349 samples were analyzed. Two hundred and eighty three samples were tested for susceptibility to SIRE and 66 to pyrazinamide by the MODS method. Seventeen (17) samples were lost after the MODS method, thus, two hundred and sixty six samples were tested for susceptibility to streptomycin, isoniazid, rifampicin and ethambutol (SIRE) and 66 to pyrazinamide, all by the MGIT method. The average duration from culture to drug susceptibilities by MODS was 12.5 (approximately 13 days, range of 7-32) for the direct method and 9.3 days (approximately 9 days, range of 6-16) for the indirect method, whilst for BACTEC MGIT 960, it was 10.1 days for culture (approximately 10 days, range 3-40), with a further 12 days to detect resistance (Table 1). Therefore, a turnaround period of 22 days was needed for DST by BACTEC MGIT 960, compared to 13 days for MODS. The difference between the turnaround time for DST by the two methods (BACTEC MGIT 960 and MODS) was statistically significant $(p<0.01)$.

Table 1. Time taken (in days) for culture and susceptibility testing.

\begin{tabular}{|c|c|c|c|c|c|c|c|}
\hline & \multicolumn{7}{|c|}{ Culture and Susceptibility Testing (MGIT, Direct and Indirect MODS) } \\
\hline & $\begin{array}{l}\text { MGIT Culture, } \\
N=283\end{array}$ & $\begin{array}{l}\text { MGIT SIRE, } \\
N=266\end{array}$ & $\begin{array}{l}\text { MGIT PZA, } \\
N=66\end{array}$ & $\begin{array}{l}\text { MGIT Susceptibility, } \\
N=266\end{array}$ & $\begin{array}{l}\text { Dir. MODS } \\
\text { SIRE, } N=283\end{array}$ & $\begin{array}{l}\text { In. MODS } \\
\text { SIRE, } N=283\end{array}$ & $\begin{array}{l}\text { MODS PZA, } \\
N=66\end{array}$ \\
\hline Maximum & 40 & 21 & 21 & 63 & 32 & 16 & 16 \\
\hline Minimum & 3 & 5 & 5 & 10 & 7 & 6 & 7 \\
\hline Average & 10.1 & 9.6 & 9.0 & 21.7 & 12.5 & 9.3 & $9 p<0.01$ \\
\hline \multicolumn{2}{|c|}{ Indeterminate13 } & 36 & 4 & NA & 0 & 0 & 0 \\
\hline
\end{tabular}

$N$ = Total number of MTB isolates tested; SIRE-streptomycin, isoniazid, rifampicin, ethambutol; PZA-pyrazinamide; In. MODS-Indirect MODS; Dir MODS-Direct MODS; Indeterminate represents samples that the MGIT instrument flagged as positive but in which no AFB was seen in their smears; thus, a possible contamination of culture was considered to have occurred, or growth was detected in only one control well for MODS-DST. Tests and controls were arranged in the BACTEC MGIT 960 instrument for a 14-day incubation period. MGIT susceptibility represents the turnaround time for the BACTEC MGIT 960 procedure (from culture through drug susceptibility testing). As part of the MGIT test protocol, about two days on average elapsed between the day the instrument flagged positive and the day the susceptibility was set.

Table 1 shows the time taken for the reading of susceptibility of $M T B$ isolates to tested antimicrobials by the various methods.

\subsection{Resistance Pattern}

Growth in the tubes or wells containing any of the antimicrobials was interpreted as a sign of resistance to that particular antimicrobial. Susceptible samples were those that showed no growth in the antimicrobial tubes or wells, even though there was growth in the corresponding control test tube or well. True resistance was defined as resistance by the two methods, and true susceptibility as those that were susceptible to the two methods. Indeterminate represents samples in which that the MGIT instrument flagged positive but no AFB was seen in their smears; thus, a possible contamination 
of culture was considered to have occurred, or growth was detected in only one control well for MODS-DST. The sensitivity, specificity, and predictive values of all the antimicrobials are displayed in Table 2.

Table 2. Resistance pattern of the antimicrobials and sensitivity, specificity, and predictive values.

\begin{tabular}{|c|c|c|c|c|c|}
\hline \multicolumn{6}{|c|}{ MGIT (For SIRE, $N=266$ and for PZA, $N=66$ ) } \\
\hline & Streptomycin & Isoniazid & Rifampicin & Ethambutol & Pyrazinamide \\
\hline Resistant, $n(\%)$ & $52(19.6)$ & $65(24.5)$ & $19(7.2)$ & $11(4.2)$ & $5(7.6)$ \\
\hline Susceptible, $n(\%)$ & $177(66.8)$ & $164(61.9)$ & $210(79.2)$ & $218(82.3)$ & $57(86.4)$ \\
\hline Indeterminate, $n(\%)$ & $36(13.6)$ & $36(13.6)$ & $36(13.6)$ & $36(13.6)$ & $4(6.0)$ \\
\hline Sensitivity (\%) & 81.3 & 82.3 & 79.2 & 57.9 & 100 \\
\hline Specificity (\%) & 100 & 97.6 & 91.3 & 99.1 & 100 \\
\hline PPV $(\%)$ & 100 & 94.2 & 86.4 & 84.6 & 100 \\
\hline NPV (\%) & 93.7 & 92.1 & 97.7 & 94.5 & 100 \\
\hline \multicolumn{6}{|c|}{ MODS (for SIRE, $N=283$ and for PZA, $N=66$ ) } \\
\hline & Streptomycin & Isoniazid & Rifampicin & Ethambutol & Pyrazinamide \\
\hline Resistant, $n(\%)$ & $79(27.9)$ & $79(27.9)$ & $23(8.1)$ & $23(8.1)$ & $4(6.1)$ \\
\hline Susceptible, $n(\%)$ & $204(72.1)$ & $193(68.2)$ & $260(91.9)$ & $260(91.9)$ & $62(93.9)$ \\
\hline Indeterminate, $n(\%)$ & 0 & 0 & 0 & 0 & 0 \\
\hline Sensitivity (\%) & 100 & 95.7 & 85.2 & 85.2 & 100 \\
\hline Specificity (\%) & 94 & 93.2 & 97.4 & 97 & 100 \\
\hline PPV $(\%)$ & 91 & 86.5 & 76.7 & 74.2 & 100 \\
\hline NPV (\%) & 100 & 98.0 & 98.5 & 99.2 & 100 \\
\hline
\end{tabular}

$N$ = Total number of MTB isolates tested; SIRE-streptomycin, isoniazid, rifampicin, ethambutol; PZA-pyrazinamide; PZA-Pyrazinamide; PPV denotes a positive predictive value and NPV a negative predictive value of the n-number of samples that were observed to be resistant, susceptible, or indeterminate.

Tested MTB isolates resistance to the primary antimicrobials by the MODS method were streptomycin $(27.9 \%)$, isoniazid $(27.9 \%)$, rifampin $(8.1 \%)$, ethambutol $(8.1 \%)$, and pyrazinamide $(6.1 \%)$. Meanwhile, those for the MGIT method were streptomycin (19.6\%), isoniazid (24.5\%), rifampicin $(7.2 \%)$, ethambutol $(4.2 \%)$, and pyrazinamide $(7.6 \%)$ (Table 2). Sensitivity of the MODS method with respect to streptomycin was $100 \%$, isoniazid (5.7\%), rifampicin $(7.2 \%)$, ethambutol $(85.2 \%)$, and pyrazinamide (100\%), while sensitivity of the BACTEC MGIT 960 method with respect to streptomycin was $81.3 \%$, rifampicin $(85.2 \%)$, and pyrazinamide (100\%) (see Table 2 ).

Concordance between MODS and MGIT for the individual antimicrobials as calculated by kappa coefficients ( $k$ ) were streptomycin (0.87), isoniazid (0.79), rifampin (0.90), ethambutol (0.63), and pyrazinamide (1.0). Kappa coefficients values that were excellent (perfect agreement) were observed for streptomycin, rifampin, and pyrazinamide (0.87-1.0) across the two tested methods, giving a nearly completely concordant result. However, low substantial agreement was apparent for ethambutol. Table 3 presents concordance and discordance of the MODS (direct and indirect) and BACTEC MGIT 960 methods.

Table 3. Concordance and discordance of the MODS (direct and indirect) and BACTEC MGIT 960 methods.

\begin{tabular}{lccccc}
\hline \multicolumn{7}{c}{ Antimicrobials } \\
\hline & Rifampin & Streptomycin & Isoniazid & Ethambutol & Pyrazinamide \\
\hline Concordance (\%) & 90 & 87 & 79 & 63 & 100 \\
Discordance (\%) & 10 & 13 & 21 & 37 & 0 \\
\hline
\end{tabular}

\section{Discussion}

The current study purposed to identify which, among the most commonly used (in Ghana) methods, can conveniently be applied at health centers located in rural areas for effective DST determination of MTB isolates. Therefore, the MODS and BACTEC MGIT 960 methods were evaluated 
for their usefulness in DST determination of $M T B$, using clinical MTB isolates. This specifically involved a comparison of the drug susceptibility outcome for MODS to BACTEC MGIT 960, and of the ease of recognizing cord formation using each of the two methods.

The study determined the susceptibility of MTB isolates to streptomycin, rifampicin, isoniazid, ethambutol, and pyrazinamide, the primary antimicrobials for TB treatment by the BACTEC MGIT method, as well as by the MODS method. Tested MTB isolates showed varying resistance and susceptibility to the primary antimicrobials by the MODS (6.1-27.9 and 68.2-93.9 respectively) and MGIT (4.2-24.5 and 61.9-86.4 respectively) methods. Meanwhile, the concordance between MODS and MGIT with respect to streptomycin was 0.87 , isoniazid (0.79), rifampin (0.90), ethambutol (0.63), and pyrazinamide (1.0).

There were, however, a few discrepancies in the results of the two methods with respect to ethambutol and, to a lesser extent, isoniazid (correlation between the two methods was $63 \%$ for ethambutol and $79 \%$ for isoniazid); however, these discrepancies were not enough to make the results obtained by the two methods statistically different. The correlation between the two methods for rifampin (90\%) and isoniazid (79\%) was in accordance with a previous work which compared MODS to the proportion method [18]. Other researchers [31,32] have found the correlation between MODS and other methods to be poor with regard to ethambutol and streptomycin.

In contrast, this study recorded levels that were higher. All the same, the extent of disagreement was not statistically significant [18]. Another study also observed disagreements between the results for rifampin and those of MODS in comparison with other methods [33]. In that study, the results were read 14 days after visible growth appeared in the drug free wells [32]. There was an earlier suggestion by Caviedes et al. [17] that the results for rifampin resistance should be confirmed with either Microplate Alamar Blue Assay (MABA) or the proportion method on Lowenstein-Jensen media.

In the current study, however, the observed differences in the results of MGIT and MODS could be attributed to any of the following three factors: inoculum size, method of reading the results, or failure rates with respect to the MGIT method. These reasons are interrelated. MGIT variation in bacterial concentration could be reduced, since the results were not manually read. On a given day of positivity, the bacterial concentrations are usually fairly the same. A manual reading of MODS makes it subjective, indicating that the concentration on the day of positivity may not be the same. Unlike the MGIT, that measures the unit of growth as well, there are no discrete colonies to count in the case of MODS, and therefore, any growth in a drug-containing well was deemed to reflect resistance to that drug. The high number of indeterminate cases (13.6\%) with respect to the MGIT method could account for the differences observed in the results of susceptibility testing by the two methods.

Regarding resistance, other studies have reported varying level of resistance in different parts of Ghana [5,31,33]. A comparative analysis of the results obtained by these studies revealed that the percentage of resistance observed in the current study was relatively higher for most of the tested antibiotics. For Streptomycin, while the current study recorded resistance of $27.9 \%$, previous studies have observed $0 \%, 9 \%$, and $23 \%[5,31,33]$. The resistance levels for the other antibiotics tested were as follows: Isoniazid: $27.9 \%$ compared to $4.2 \%$ and $23 \%$ [4,31], Rifampin: $8.1 \%$ compared to $0 \%$ and $0.7 \%$ [5,33], Ethambutol: $8.1 \%$ compared to $0 \%$ [5,33], and Pyrazinamide: $6.1 \%$ compared to $0 \%$ and $0.5 \%[5,33]$. MDR-TB was also recorded to be $6.7 \%$ for the current study, compared to $0 \%$ and $2.2 \%$ in earlier studies [5,33]. It is noteworthy that the current study observed resistance in the two methods for more antimicrobials (6), compared to previous studies by other investigators in Ghana $[5,31,33]$.

The marked increase in resistance to virtually all the primary antimicrobials within the study period represents a major health concern. For instance, the isoniazid resistance of $27.9 \%$ observed in this study could have negative implications for TB patients, since such resistance is associated with poor clinical outcomes [34], with recent guidelines already having been issued for a global rollout of an isoniazid-resistant regimen [35]. Increases in resistance have been recorded at other locations near Ghana. N'Guessan et al. [36] reported higher prevalence of multidrug-resistant tuberculosis in Côte d'Ivoire from 1995 to 2016. It has also been shown in a systematic review on the prevalence of DR-TB 
in Nigeria and Ethiopia that the current WHO estimate for new and previously-treated TB patients for these countries was low $[37,38]$, providing evidence that the burden of DR-TB in high-TB-burden countries, especially in Africa, is probably higher than what has been predicted [4].

Considering the individual antimicrobials separately, there were rises in resistance to isoniazid (5.0 to $6.9 \%$ ) and rifampicin (1.0 to $1.2 \%$ ), but not to ethambutol and pyrazinamide. In Belarus, out of $163 \mathrm{~TB}$ isolates that were tested against the antibiotics, 42 (68.5\%) were identified as being resistant to isoniazid, rifampicin, and streptomycin, and eight (28\%) were resistant to ethambutol [34]. Monoresistance to isoniazid was observed in four (14\%) isolates [39]. In this study, the reading of cord formation via the MODS method was done with ease. This is an important criterion; therefore, the MODS method is hereby endorsed, as it is less technically challenging, and hence, can easily be used in a clinical setting with limited facilities, especially in rural parts of in Ghana.

\section{Conclusions}

This study determined that the MODS method is efficient in determining the susceptibility of MTB isolates to primary antibiotics for TB treatment. This method was observed to be cost effective and easily applicable in health centers of TB-endemic, rural communities in Ghana. It could therefore be introduced alongside the BACTEC MGIT system, with little training being required due to its ease of use.

Author Contributions: Conceptualization, E.O. and M.J.N.; Formal analysis, E.O.; Investigation, E.O. and M.J.N.; Methodology, E.O. and M.J.N.; Resources, E.O. and M.J.N.; Supervision, M.J.N.; Validation, E.O. and M.J.N.; Visualization, E.O.; Writing-original draft, E.O. and M.J.N.; Writing—review \& editing, E.O. and M.J.N. All authors have read and agreed to the published version of the manuscript.

Funding: This research received no external funding.

Acknowledgments: The authors wish to thank all the staff of the Departments of Medical Laboratory Sciences and Medical Microbiology (all of SBAHS) for their help. We also wish to thank all TB patients (subjects) who participated in the study. We also acknowledge the late Nsiah Owusu Poodoh for his immense contribution in most activities of this work.

Conflicts of Interest: The authors declare no conflict of interest.

\section{References}

1. Glaziou, P.; Sismanidis, C.; Floyd, K.; Raviglione, M. Global epidemiology of tuberculosis. Cold Spring Harb. Perspect. Med. 2014, 5, a017798. [CrossRef] [PubMed]

2. Sandhu, G.K. Tuberculosis: Current situation, challenges and overview of its control programs in India. J. Glob. Infect. Dis. 2011, 3, 143-150. [CrossRef] [PubMed]

3. Mohajan, H.K. Poverty and Economic Development of Kenya. Int. J. Inf. Technol. Bus. Manag. 2013, 18, 72-82.

4. Global Tuberculosis Report 2019; World Health Organization: Geneva, Switzerland, 2019; Licence: CC BY-NC-SA 3.0 IGO; Available online: https://www.who.int/tb/publications/global_report/en/ (accessed on 20 December 2019).

5. Owusu-Dabo, E.; Adjei, O.; Meyer, C.; Horstmann, R.D.; Enimil, A.; Kruppa, T.F.; Bonsu, F.; Browne, E.N.; Chinbuah, M.A.; Osei, I.; et al. Mycobacterium tuberculosis drug resistance, Ghana. Emerg. Infect. Dis. 2006, 12, 1171-1172. [CrossRef]

6. Grange, J.M.; Zumla, A. The global emergency of tuberculosis: What is the cause? J. R. Soc. Health 2002, 122, 78-81. [CrossRef]

7. Frieden, T.R.; Sterling, T.R.; Munsiff, S.S.; Watt, C.J.; Dye, C. Tuberculosis. Lancet 2003, 362, 887-899. [CrossRef]

8. Muralidhar, S.; Srivastava, L. Evaluation of three methods to determine the antimicrobial susceptibility of Mycobacterium tuberculosis. Indian J. Med Res. 2004, 120, 463-467.

9. Cui, Z.; Wang, J.; Lu, J.; Huang, X.; Zheng, R.; Hu, Z. Evaluation of Methods for Testing the Susceptibility of Clinical Mycobacterium tuberculosis Isolates to Pyrazinamide. J. Clin. Microbiol. 2013, 51, 1374-1380. [CrossRef] 
10. Gopinath, K.; Singh, S. Multiplex PCR assay for simultaneous detection and differentiation of Mycobacterium tuberculosis, Mycobacterium avium complexes and other Mycobacterial species directly from clinical specimens. J Appl. Microbiol. 2009, 107, 425-435. [CrossRef]

11. Naveen, G.; Peerapur, B.V. Comparison of the Lowenstein-Jensen Medium, the Middlebrook 7H10 Medium and MB/BacT for the Isolation of Mycobacterium Tuberculosis (MTB) from Clinical Specimens. J. Clin. Diagn. Res. 2012, 6, 1704-1709. [CrossRef]

12. Forbes, B.A.; Hall, G.S.; Miller, M.B.; Novak, S.M.; Rowlinson, M.C.; Salfinger, M.; Somoskövi, A.; Warshauer, D.M.; Wilson, M.L. Practice Guidelines for Clinical Microbiology Laboratories: Mycobacteria. Clin. Microbiol. 2018, 31, e00038-17. [CrossRef] [PubMed]

13. Ridderhof, J.C.; Deun, A.V.; Kam, K.M.; Narayanan, P.R.; Azi, M.A. Roles of laboratories and laboratories systems in effective tuberculosis programmes. Bull. World Health Organ. 2007, 85, 345-359. [CrossRef] [PubMed]

14. Paramasivan, C.N. Two speedier phenotypic methods on drugs susceptibility testing of mycobacterium. Indian J. Med. Res. 2004, 120, 440-441. [PubMed]

15. Moore, D.A.; Evans, C.A.; Gilman, R.H.; Caviedes, L.; Coronel, J.; Vivar, A.; Sanchez, E.; Piñedo, Y.; Saravia, J.C.; Salazar, C.; et al. Microscopic observation drug susceptibility assay for the diagnosis of TB. $N$ Engl. J. Med. 2006, 355, 1539-1550. [CrossRef] [PubMed]

16. Kadam, M.; Govekar, A.; Shenai, S.; Sadani, M.; Salvi, A.; Shetty, A.; Rodrigues, C. Can cord formation in BACTEC MGIT 960 medium be used as a presumptive method for identification of M. tuberculosis complex? Indian J. Tuberc. 2010, 57, 75-79.

17. Caviedes, L.; Lee, T.S.; Gilman, R.H.; Sheen, P.; Spellman, E.; Lee, E.H.; Berg, D.E.; Montenegro-James, S. Rapid, efficient detection and drug susceptibility testing of Mycobaterium tuberculosis in sputum by microscopic observation of broth cultures. J. Clin. Microbiol. 2000, 3, 1203-1208. [CrossRef]

18. Park, W.G.; Bishai, W.R.; Chaisson, R.E.; Dorman, S.E. Performance of the microscopic observation drug susceptibility assay in drug susceptibility testing for Mycobacterium tuberculosis. J. Clin. Microbiol. 2002, 12, 4750-4752. [CrossRef]

19. Oberhelman, R.A.; Soto-Castellares, G.; Caviedes, L.; Castillo, M.E.; Kissinger, P.; Moore, D.A.J.; Evans, C.; Gilman, R.H. Improved recovery of Mycobacterium tuberculosis from children using the microscopic observation drug susceptibility method. Pediatrics 2006, 188, e100-e106. [CrossRef]

20. Tayyab, N.; Zaman, G.; Satti, L.; Ikram, A.; Gardezi, A.H.; Khadim, M.T. Direct Susceptibility Testing on MGIT 960 TB System: A Rapid Method for Detection of Drug Resistant Tuberculosis. J. Coll. Physicians Surg. Pak. 2018, 28, 590-593. [CrossRef]

21. Ewer, K.; Deeks, J.; Alvarez, L.; Bryant, G.; Waller, S.; Andersen, P.; Monk, P.; Lalvani, A. Comparison of T-cell-based assay with tuberculin skin test for diagnosis of Mycobacterium tuberculosis infection in a school tuberculosis outbreak. Lancet 2003, 9364, 1168-1173. [CrossRef]

22. Ryu, Y.J. Diagnosis of Pulmonary Tuberculosis: Recent Advances and Diagnostic Algorithms. Tuberc. Respir Dis. 2015, 2, 64-71. [CrossRef] [PubMed]

23. Nyendak, M.D.; Lewinsohn, D.A.; Lewinsohn, D.M. New Diagnostic Methods for Tuberculosis. Curr. Opin. Infect. Dis. 2009, 22, 174-182. [CrossRef] [PubMed]

24. WHO. Global Tuberculosis Report. Available online: http://www.who.int/tb/publications/global_report/en/ (accessed on 12 May 2017).

25. Kent, P.T.; Kubica, G.P. Public Health Microbiology. A Guide for The Level Ill Laboratory; Centers for Disease Control: Atlanta, GA, USA, 1985.

26. Kassaza, K.; Orikiriza, P.; Llosa, A.; Bazira, J.; Nyehangane, D.; Page, A.-L.; Boum, Y. Lowenstein-Jensen Selective Medium for Reducing Contamination in Mycobacterium tuberculosis Culture. J. Clin. Microbiol. 2014, 52, 2671-2673. [CrossRef] [PubMed]

27. Diriba, G.; Kebede, A.; Yaregal, Z. Performance of Mycobacterium Growth Indicator Tube BACTEC 960 with Lowenstein-Jensen method for diagnosis of Mycobacterium tuberculosis at Ethiopian National Tuberculosis Reference Laboratory, Addis Ababa, Ethiopia. BMC Res. Notes 2017, 10, 181. [CrossRef] [PubMed]

28. Musa, R.H.; Ambroggi, M.; Souto, A.; Angeby, K.A. Drug susceptibility testing of Mycobacterium tuberculosis by a nitrate reductase assay applied directly on microscopy-positive sputum samples. J. Clin. Microbiol. 2005, 43, 3159-3161. [CrossRef] [PubMed] 
29. World Health Organisation. Guidelines for Surveillence of Drug Resistance in Tuberculosis. WHO/TB/2003.320-WHO/CDS/CSR/RMD/2003.3. 2003. Available online: http://whqlibdoc.who.int/ publications/2003/9241546336.pdf (accessed on 22 March 2011).

30. McHugh, M.L. Interrater reliability: The kappa statistic. Biochem. Med. 2012, 22, 276-282. [CrossRef]

31. Lawn, S.D.; Frimpong, E.H.; Al-Ghusein, H.; Acheampong, J.W.; Uttley, A.H.; Butcher, P.D.; Griffin, G.E. Pulmonary tuberculosis in Kumasi Ghana: Presentation, drug resistant, molecular epidemiology and outcome of treatment. West Afr. J. Med. 2001, 20, 92-97.

32. Mello, F.C.; Arias, M.S.; Rosales, S.; Marsico, A.G.; Pavón, A.; Alvarado-Gálvez, C.; Pessôa, C.L.; Pérez, M.; Andrade, M.K.; Kritski, A.L.; et al. Clinical evaluation of the microscopic observation drug susceptibility assay for detection of Mycobacterium tuberculosis resistance to isoniazid or rifampin. J. Clin. Microbiol. 2007, 45, 3387-3389. [CrossRef]

33. Van Der Werf, T.S.; Van Der Graaf, W.T.; Groothuis, D.G.; Knell, A.J. Mycobacterium ulcerans infection in Ashanti region, Ghana. Trans. R. Soc. Trop. Med. Hyg. 1989, 83, 410-413. [CrossRef]

34. Gegia, M.; Winters, N.; Benedetti, A.; van Soolingen, D.; Menzies, D. Treatment of isoniazid-resistant tuberculosis with first-line drugs: A systematic review and meta-analysis. Lancet Infect. Dis. 2017, 17, 223-234. [CrossRef]

35. Bouton, T.C.; Forson, A.; Kudzawu, S.; Zigah, F.; Jenkins, H.; Bamfo, T.D.; Carter, J.; Jacobson, K.; Kwara, A. High mortality during tuberculosis retreatment at a Ghanaian tertiary center: A retrospective cohort study. Pan. Afr. Med. J. 2019, 13, 111. [CrossRef] [PubMed]

36. N'Guessan, K.; Ouassa, T.; Dean, A.S.; Alagna, R.; Adagra, G.D.; Ibode, V.; Cirillo, D.M.; Kouakou, J. Multidrug-Resistant Tuberculosis in Côte d'Ivoire from 1995 to 2016: Results of National Surveys. Eur. J. Microbiol. Immunol. (Bp). 2018, 16, 91-94. [CrossRef] [PubMed]

37. Onyedum, C.C.; Alobu, I.; Ukwaja, K.N. Prevalence of drug-resistant tuberculosis in Nigeria: A systematic review and meta-analysis. PLoS ONE 2017, 12, e0180996. [CrossRef]

38. Weldegebreal, S.; Mebrahtu, T. Anti-tuberculosis drug resistance in Ethiopia: Systematic review. Int. J. Tuberc. Lung Dis. 2017, 21, 18-22. [CrossRef] [PubMed]

39. Bostanabad, S.Z.; Titov, L.P.; Bahrmand, A.; Nojoumi, S.A. Detection of mutation in isoniazid-resistant Mycobacterium tuberculosis isolates from tuberculosis patients in Belarus. Indian J. Med. Microbiol. 2008, 26, 143-147. [CrossRef] [PubMed] 